\title{
The value hypothesis and acquisition of preference in concurrent chains
}

\author{
RANDOLPH C. GRACE \\ University of Canterbury, Christchurch, New Zealand
}

\begin{abstract}
Experiment 1 compared the acquisition of initial- and terminal-link responding in concurrent chains. The terminal-link schedules were fixed interval (FI) $10 \mathrm{sec}$ and FI $20 \mathrm{sec}$, but some presentations were analogous to no-food trials in the peak procedure, lasting $60 \mathrm{sec}$ with no reinforcement delivery. Pigeons completed a series of reversalsin which the schedules signaled by the terminal-link stimuli (red and green on the center key) were changed. Acquisition of temporal control of terminal-link responding (as measured by peak location on no-food trials) was more rapid than acquisition of preference in the initial links. Experiment 2 compared acquisition in concurrent chains, using the typical procedure in which the terminallink schedules are changed with a novel arrangement in which the initial-link key assignments were changed while the terminal-linkschedules remained the same. Acquisition of preference was faster in the latter condition, in which the terminal-link stimulus-reinforcer relations were preserved. These experiments provide the first acquisition data that support the view that initial-link preference is determined by the values of the terminal-link stimuli.
\end{abstract}

The concurrent-chains procedure has been used to study choice between stimuli that have acquired differential value. In a typical arrangement, a pigeon is confronted with two side keys lighted white in a three-key operant chamber (see Figure 1). This signals the choice phase, or initial links. Concurrent variable-interval(VI) VI schedules operate during the initial links, so that occasionally and unpredictably, a response to either initial link extinguishes the side keys and illuminates the center key. This signals the outcome phase, or terminal link. The color of the center key depends on the location of the preceding initial-link response; for example, the left initial link produces a red center key, and the right initial link produces a green center key. Responding during the red or green terminal links results in access to food according to different reinforcement schedules, after which the initial links are reinstated.

From the beginning, investigators assumed that choice during the initial links corresponded to the relative strength or value of the terminal-link stimuli as conditioned reinforcers (e.g., Autor, 1960; Herrnstein, 1964). For example, if the red and green terminal links are fixed interval (FI) 10 -sec and FI 20-sec schedules, respectively, as in Figure 1, the usual result is that pigeons respond relatively more to the initial link leading to the FI 10-sec terminal link (Killeen, 1970). The red key is said to have relatively greater value as a conditionedreinforcer because it signals a shorter delay to reinforcement.

Some of these data were presented at the annual meeting of the Society for the Quantitative Analyses of Behavior, Washington, DC, May 2000. I thank Tony Nevin and Orn Bragason for helpful comments. Address correspondence to R. C. Grace, University of Canterbury, Department of Psychology, Private Bag 4800, Christchurch, New Zealand (e-mail: r.grace@psyc.canterbury.ac.nz).
Although there has been some controversy regarding the influence of overall initial- and terminal-link durations on terminal-link value (i.e., the temporal context of choice; see Grace \& Savastano, 2000), the assumption that terminallink value is a primary determiner of responding during the initial links has been common to all models of concurrent chains (e.g., Davison \& Temple, 1973; Fantino, 1969; Herrnstein, 1964; Killeen, 1982; Squires \& Fantino, 1971; Vaughan, 1985). Grace $(1994,1996)$ showed that the results of many studies that have parametrically varied reinforcement rate, immediacy, and magnitude during the terminal links support an extension of the matching law to concurrent chains, in which relative response rate during the initial links matches the relative value of the terminal-link stimuli. The ability of a model to explain a high percentage of variance provides some support, albeit indirect, for the assumptions regarding terminal-link value (Grace, 1996).

However, despite the empirical success of these models, it is important to note that terminal-link value is a theoretical construct, so the assumption that choice responding is mediated by differential value is a hypothesis, which for present purposes may be termed the value hypothesis. Prior research has studied steady-state performance almost exclusively. For example, a typical experiment (e.g., Alsop \& Davison, 1988) might include a large number of conditions over which terminal-link reinforcement parameters are varied. In each condition, training continues until preference reaches a stable asymptote (often 20 sessions or more). Models are then evaluated in terms of the proportion of variance accounted for in preference across conditions. As a consequence of the emphasis on molar, steadystate performance, little is known about acquisition in concurrent chains - in particular, whether such data would support the view that choice is determined by the value of 


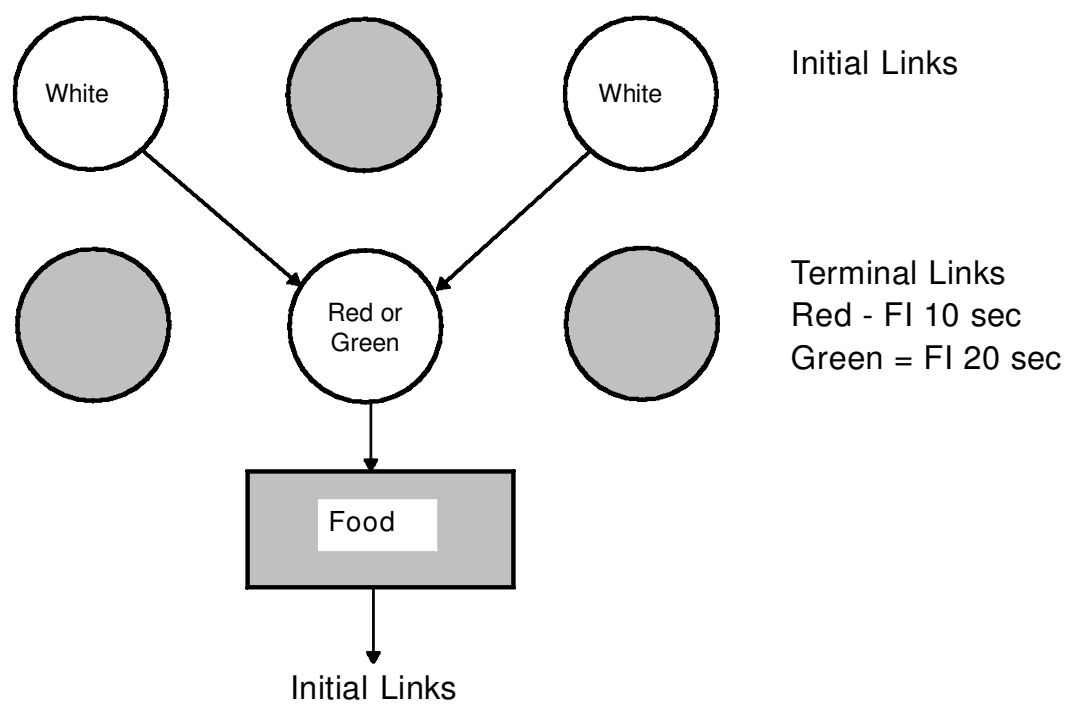

Figure 1. Schematic diagram of a typical concurrent-chains procedure. Concurrent variable interval (VI) 30-sec VI 30 -sec schedules operate during the initial links. Responses to the white keys during the initial links occasionally result in the presentation of one of two mutually exclusive terminal links (red and green keys). Terminal-link responses produce access to food according to separate reinforcement schedules (FI $10 \mathrm{sec}$ for red, FI $20 \mathrm{sec}$ for green), after which the initial links are reinstated.

the terminal-link stimuli. In fact, the available evidence paints a more complicated picture than the value hypothesis would suggest.

Grace and Nevin (1999) used a novel concurrent-chains procedure that allowed simultaneous measures of choice in the initial links and estimates of expected terminal-link delays to reinforcement to be obtained. To accomplish this, Grace and Nevin (1999) integrated a peak procedure within concurrent chains. The peak procedure (Catania, 1970; Roberts, 1981) is a discrete-trials FI schedule in which some trials extend well past the FI value and end without reinforcement. When responding on these no-food trials is aggregated, a typical pattern emerges: Response rate as a function of elapsed time within the trial is approximately a normal distribution with a mode (i.e., peak) corresponding to the FI value. The peak procedure has been extensively used in research on timing, with the location of the peak interpreted as the animal's estimate of the time of reinforcement delivery.

In Grace and Nevin's (1999) procedure, $75 \%$ of the terminal-link presentations were FI schedules that ended in reinforcement, and 25\% were no-food or "peak trials" that lasted for three times the FI value and ended without reinforcement. After baseline training with FI 10-sec FI 20 -sec terminal links, the pigeons were switched to a simple peak procedure with FI 40-sec and FI 20-sec schedules. The same terminal-link stimuli were used, so this new condition constituted, in effect, revaluation training (Williams, Ploog, \& Bell, 1995; cf. Adams \& Dickinson, 1981). The critical test occurred when the pigeons were returned to concurrent chains. Although subjects continued to time the 40 -sec and 20-sec delays accurately, as indicated by ongoing peak-trial responding, preference during the initial links changed only slowly over sessions to favor the FI $20 \mathrm{sec}$. Thus, according to ongoing peak-trial responding, the FI 20-sec stimulus must have had greater value; yet preference during the initial links continued to favor the FI $40 \mathrm{sec}$. Grace and Nevin (1999) argued that the dissociation between initial- and terminal-link responding posed a challenge to the view that choice in the initial links was directly mediated by terminal-link value, as well as to theories that maintain that choice responding is based on a representation of terminal-link delays to reinforcement (Gallistel \& Gibbon, 2000; Gibbon, Church, Fairhurst, \& Kacelnik, 1988). For present purposes, note that value and representation are fundamentally similar in that both are theoretical constructs.

The present research tested two predictions of the value hypothesis. Experiment 1 compared acquisition of temporal control of terminal-link responding on peak trials with acquisition of initial-link preference. We assume that responding during the peak trials provides a direct measure of the expected time to terminal-link reinforcement, which determines value. If preference is mediated by terminallink value, shifts in temporal control of terminal-link responding should occur prior to or concurrently with shifts in preference. The value hypothesis also predicts that acquisition of initial-link preference should be faster if the terminal-link stimuli continue to signal the same reinforcement schedules than if those reinforcement schedules are changed. This prediction was tested in Experiment 2.

\section{EXPERIMENT 1}

Experiment 1 used a combined concurrent-chains/peak procedure (Grace \& Nevin, 1999) with FI 10-sec and FI 
20 -sec terminal links. Across successive conditions, the location of the initial link that produced the FI 10-sec terminal link was changed. The terminal links were equivalent to trials in a peak procedure. Food was available on $75 \%$ of the presentations of each terminal link according to the FI schedule. On the remaining $25 \%$ of the presentations, food was not available, and the terminal link lasted for $60 \mathrm{sec}$ and ended independently of responding. Thus, in each session, two measures of the effects of terminal-link reinforcement were obtained: initial-link preference and the peak locations of response distributions during no-food terminal-link trials. The critical issue was to compare acquisition rates for initial- and terminal-link responding.

\section{Method}

Subjects. Four White Carneau pigeons, numbered 123, 125, 139, and 154 , participated as subjects and were maintained at $85 \%$ of their ad-lib. weight $\pm 15 \mathrm{~g}$. All the birds had previous experience with concurrent chains but were naive as to timing procedures. They were housed individually in a vivarium with a 12:12-h light:dark cycle (lights on at $7 \mathrm{am}$ ). Water and grit were available continuously in the home cages.

Apparatus. Four standard three-key operant chambers were used. The chambers were $35 \mathrm{~cm}$ in length $\times 35 \mathrm{~cm}$ in width $\times 35 \mathrm{~cm}$ in height, with keys located $26 \mathrm{~cm}$ above the floor. The keys could be illuminated with red, white, or green light. There was a houselight $7 \mathrm{~cm}$ above the center key for general illumination and a grain magazine with a $6 \mathrm{~cm} \times 5 \mathrm{~cm}$ opening located $13 \mathrm{~cm}$ below the center key. The magazine was illuminated when wheat was made available. A force of approximately $0.10 \mathrm{~N}$ was required to operate each key, and effective responses produced an audible feedback click. Chambers were enclosed in sound-attenuating boxes and were fitted with ventilation fans for masking extraneous noises. The experiment was controlled and data were collected with a MED-PC system interfaced to an IBM-compatible microcomputer located in an adjacent room.

Procedure. Because the subjects were experienced, training began on a concurrent-chains/ peak procedure (Grace \& Nevin, 1999). Sessions consisted of 72 initial- and terminal-link cycles. At the start of a cycle, the left and right keys were illuminated white to signal the initial links, or choice phase, of the procedure. A terminal-link entry was assigned randomly to either the left or the right key, with the constraint that out of every eight terminal links, four were assigned to the left key and four to the right key. Thus, the initial links used interdependent scheduling (i.e., forced choice; Stubbs \& Pliskoff, 1969). A response produced an entry into a terminal link if (1) it was to the preselected key, (2) an interval selected from a VI 8-sec schedule at the start of the cycle had timed out, and (3) a 1-sec changeover delay (COD) was satisfied, (i.e., at least $1 \mathrm{sec}$ had elapsed following a changeover to the key for which terminal-link entry was arranged). The VI $8 \mathrm{sec}$ contained 12 intervals constructed from an arithmetic progression, $a, a+d, a+2 d, \ldots$, where $a$ equals one twelfth and $d$ equals one sixth the schedule value. The intervals were sampled randomly without replacement. The initial-link schedule did not begin timing until the first response of the cycle had been made.

Entry into a terminal link was signaled by red or green illumination of the center key, together with extinguishing of the side keys. Terminal links that were produced by responses to the left initial link were signaled by red on the center key; those that were produced by right initial-link responses were signaled by green. The terminal-link schedules were always FI $10 \mathrm{sec}$ and FI $20 \mathrm{sec}$ (assigned to red and green or vice versa, depending on condition), and each was presented 36 times per session. Of the 36 presentations of each terminal link, 27 were food trials that ended in reinforcement following the first response after the FI schedule had elapsed. Reinforcement consisted of a 3-sec access to grain, during which all lights in the chamber were extinguished. A 5-sec limited hold operated on food trials so that, if a response had not been made within $5 \mathrm{sec}$ after the FI had elapsed, the terminal link was canceled and the initial links were reinstated. The other 9 presentations were peak trials that lasted for $60 \mathrm{sec}$ (for both FI 10-sec and FI 20-sec terminal links) and ended without reinforcement. After each terminal link ended, the side keys were illuminated white, signaling the initial links, and the next cycle began. The houselight remained on at all times, except during reinforcement.

The sequence of experimental conditions is listed in Table 1 for all the subjects. A successive-reversa 1 design was used in which the position of the initial link leading to the FI 10-sec terminal links was changed across conditions. The first two conditions lasted for 27 and 25 sessions, respectively. Although responding had typically stabilized after 15-20 sessions, Conditions 3 and 4 were maintained for 40 and 53 sessions, respectively, so that variability in different measures of performance at asymptote could be assessed.

Sessions were generally conducted each day at approximately the same time. There was a 1-week gap between Sessions 22 and 23 of Condition 3, but this had no discernable effect on the pigeons' behavior.

Measure of peak location. The location of the response peak was obtained by using the trimmed median method of Cheng and Roberts (1991). First, a cumulative response distribution was generated, and the bin that contained the median response was located. The median time was then obtained by linear interpolation. A new cumulative response distribution was then generated after excluding all responses that occurred after twice the median. A median was calculated for the new distribution, and the process was iterated until successive medians stopped changing. The final median was taken as an estimate of the peak time. The advantage of this nonparametric procedure is that it provides a reliable measure of peak location from single-session data (Grace \& Nevin, 1999).

All significance tests used the .05 level.

\section{Results}

The primary dependent variables analyzed were preference in the initial links (defined as the proportion of re-

Table 1

Order of Conditions in Experiment 1, With Number of Sessions of Training

\begin{tabular}{|c|c|c|c|c|c|c|c|c|}
\hline \multirow[b]{2}{*}{ Condition } & \multicolumn{2}{|c|}{ Bird 123} & \multicolumn{2}{|c|}{ Bird 125} & \multicolumn{2}{|c|}{ Bird 139} & \multicolumn{2}{|c|}{ Bird 154} \\
\hline & Order & No. & Order & No. & Order & No. & Order & No. \\
\hline 1 & FI 10 FI 20 & 27 & FI 10 FI 20 & 27 & FI 20 FI 10 & 27 & FI 20 FI 10 & 27 \\
\hline 2 & FI 20 FI 10 & $25^{*}$ & FI 20 FI 10 & $25^{*}$ & FI 10 FI 20 & 25 & FI 10 FI 20 & $25^{*}$ \\
\hline 3 & FI 10 FI 20 & $40 *$ & FI 10 FI 20 & $40 *$ & FI 20 FI 10 & 40 & FI 20 FI 10 & $40^{*}$ \\
\hline 4 & FI 20 FI 10 & $52 *$ & FI 20 FI 10 & $53 *$ & FI 10 FI 20 & 53 & FI 10 FI 20 & $53^{*}$ \\
\hline
\end{tabular}

Note-The two fixed-interval (FI) values shown for each condition represent the terminal-link schedules associated with the left and right initial links, respectively. *Acquisition curve was obtained in that condition. 
sponses to the left key) and a measure of temporal control derived from responding on no-food trials during the terminal links. This measure was based on the assumption that peak location corresponds to the expected time to reinforcement in the terminal link and was defined as the relative expected immediacy of reinforcement, (1/PeakL)/ $+1 /$ PeakR), where PeakL and PeakR are the medians (i.e., peak locations) of the response distributions during nofood trials for the left and the right terminal links.

All the subjects, with the exception of Bird 139, responded consistently in the terminal links, so that reinforcers were rarely canceled under the 5 -sec limited hold. Because Bird 139 failed to earn more than a single reinforcer on the FI 20-sec terminal link in the first four sessions of Conditions 2-4, its acquisition data were discarded. However, Bird 139 eventually responded consistently on the FI 20 -sec in each condition, so the steadystate data for this bird were included.

Steady-state data are presented in Figure 2, which illustrates changes in initial- and terminal-link responding across successive conditions. Data for each condition and subject were averaged across the last five sessions and then were averaged across subjects. Consistent with previous results (e.g., Killeen, 1970), preference was more extreme than (i.e., overmatched) the FI schedule ratio. The geometric mean of the preference ratio across conditions was 4.69:1, as compared with an FI schedule ratio of 2:1. In contrast, relative expected immediacy approximately matched the FI schedule ratio, since average peak locations $(11.58$ and $22.52 \mathrm{sec}$ ) were close to the FI values. The geometric mean of the expected immediacy ratios was $2.03: 1$. This shows that the pigeons timed the reinforce- ment delays accurately, consistent with previous studies using peak procedures with two delays presented in each session (e.g., Beam, Killeen, Bizo, \& Fetterman, 1998; Grace \& Nevin, 2000).

Figure 3 shows the full course of acquisition for initiallink preference and terminal-link expected immediacy measures. Data are given for the first 22 sessions of each condition and are averaged across conditions and subjects. For both initial- and terminal-link measures, relative responding shifted toward the FI $10 \mathrm{sec}$ when the terminal links were reversed, eventually reaching new asymptotic levels. The individual data corresponding to Figure 3 were entered into a repeated measures analysis of variance (ANOVA), with measure (initial or terminal link) and session as factors. The interaction between measure and session was significant $[F(21,63)=6.84]$, as was the main effect of session $[F(21,63)=17.46]$. However, the effect of measure was not significant $[F(1,3)=0.05$, n.s.]. The cause of the interaction was the intersection of initial-and terminal-link acquisition curves.

The major goal of Experiment 1 was to compare acquisition rates for initial- and terminal-link responding. However, Figure 3 shows that the extent of change was greater for preference than for expected immediacy. This complicates the comparison, because it suggests that a greater change in the absolute level of responding may not represent faster acquisition, measured as a proportion of eventual change. Thus, two analyses were conducted to provide a comparison that was not confounded by differences in absolute change.

First, a hyperbolic function was fitted to the groupmean data in Figure 3. This function, $y=a x /(b+x)$,

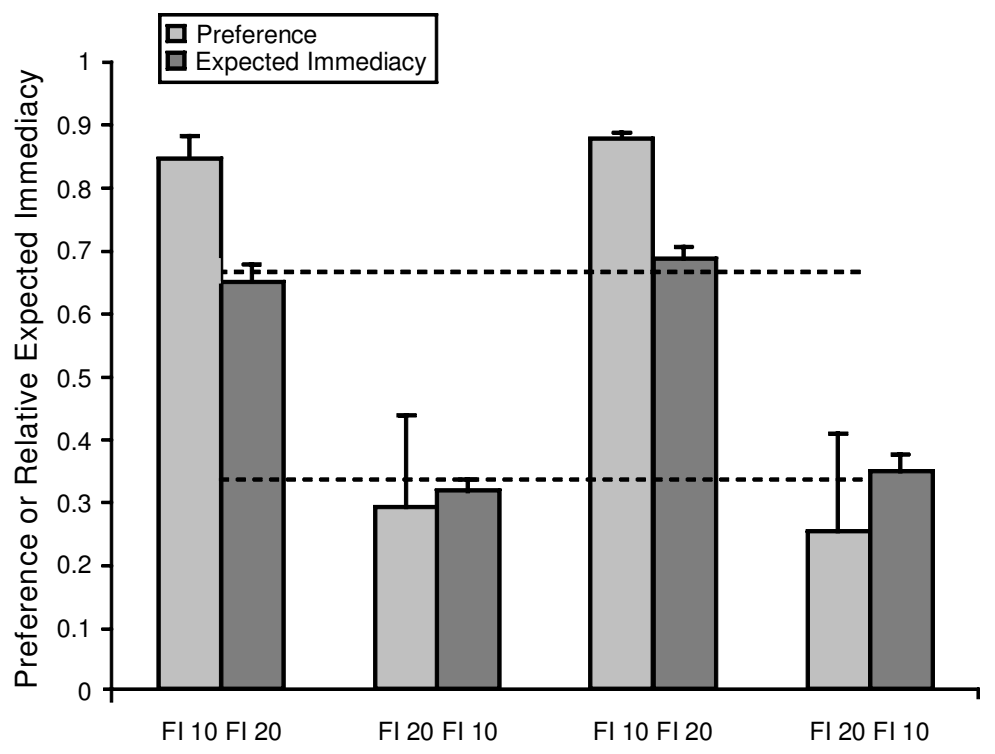

Figure 2. Steady-state preference (initial links, light gray bars) and relative expected immediacy (terminal links, dark gray bars) for each condition in Experiment 1 . The dashed line indicates matching to relative reinforcement rate, which was 2:1 or 1:2 in all conditions. Data are averaged across subjects; error bars indicate one standard error. 


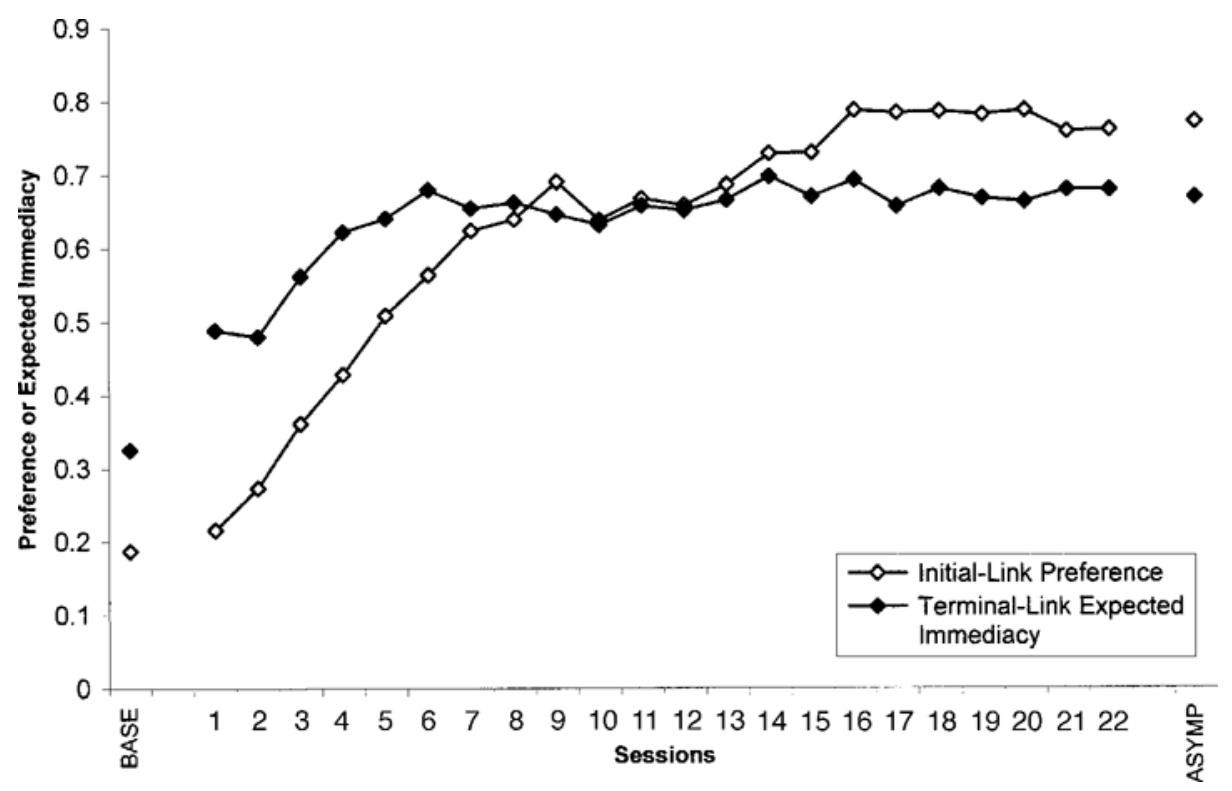

Figure 3. The full course of acquisition for preference (unfilled symbols) and relative expected immediacy (filled symbols). The points marked BASE on the abscissa indicate the asymptotes from the previous condition (i.e., baseline), in which the terminal links were FI 20 sec FI 10 sec. Data for the following condition, in which the terminal links were changed to FI $10 \mathrm{sec}$ FI $20 \mathrm{sec}$, are then shown (22 sessions). The points marked ASY MP indicate the average over the last 5 sessions. Data are averaged across conditions and subjects.

where $x$ is session number and $a$ and $b$ are fitted parameters, has been found to describe acquisition of preference for concurrent schedules (Mazur, 1992). This function accounted for $97 \%$ and $84 \%$ of the variance in the initial- and terminal-link acquisition data, respectively. To compare the rates of acquisition, we then used the fitted parameters to determine the values of $x$ for which responding had reached a criterion of $75 \%$ of the eventual asymptote. The values of $x$ were as follows: initial links, 8.45 sessions; terminal links, 2.98 sessions. Thus, initiallink preference required approximately 5.5 additional sessions, as compared with expected immediacy, to reach the $75 \%$ criterion.

In the second analysis, the confounding factor of extent of change was eliminated by normalizing both initial-and terminal-link measures. The change in each session, relative to baseline, was expressed as a proportion of the eventual change - that is, $\left(x_{i}-x_{n}\right) /\left(x_{n+1}-x_{n}\right)$, where $x_{n}$ and $x_{n+1}$ are asymptotic measures for conditions $n$ and $n+1$, and $x_{i}$ is the measure for the $i$ th postreversal session in condition $n+1$. This measure effectively places initial- and terminal-link responding on a common scale, where 0 indicates no change from previous baseline and 1 means that asymptotic performance has been reached.

Figure 4 shows acquisition measures for both initialand terminal-link responding over the first five sessions of the three reversals (Conditions 2-4). The first five sessions were used for this analysis because early postreversal sessions should be maximally sensitive to differences in acquisition rate. The individual data were entered into a repeated measures ANOVA, with measure (initial vs. terminal link), replication, and session as factors. As is predicted by the value hypothesis, normalized acquisition scores for terminal-link responding were significantly greater than the corresponding scores for initial-link responding $[F(1,2)=77.17]$. The effect of session was also significant $[F(4,8)=36.67]$. No other main effects or interactions were significant, although the measure $\times$ replication interaction approached significance $(p=.07)$, and inspection of Figure 4 suggests a tendency for terminallink acquisition measures to increase across replications.

To provide a more detailed understanding of the rapid transition in terminal-link expected immediacy, data were analyzed separately for the FI 10-sec and the FI 20-sec schedules. Figure 5 shows the median peak time measures for both schedules, averaged across conditions and subjects. A repeated measures ANOVA yielded a significant effect of schedule $[F(1,3)=110.49]$ and a schedule $\times$ session interaction $[F(21,63)=10.88]$, but no main effect of session $[F(21,63)=0.93$, n.s. $]$. The interaction implies that a reliable difference in peak medians did not emerge until the fourth or fifth session, by which time the asymptotic values had largely been reached. The data in Figure 5 suggests that the transition from FI 10 to FI 20 occurs more rapidly than that from FI 20 to FI 10 . This would appear to conflict with the results of Higa and colleagues, who found that waiting times for both rats and pigeons under fixed-time schedules of food delivery show more rapid adjustment to abrupt decreases than to increases in the interfood interval (Higa, 1996; Higa, Thaw, \& Stad- 

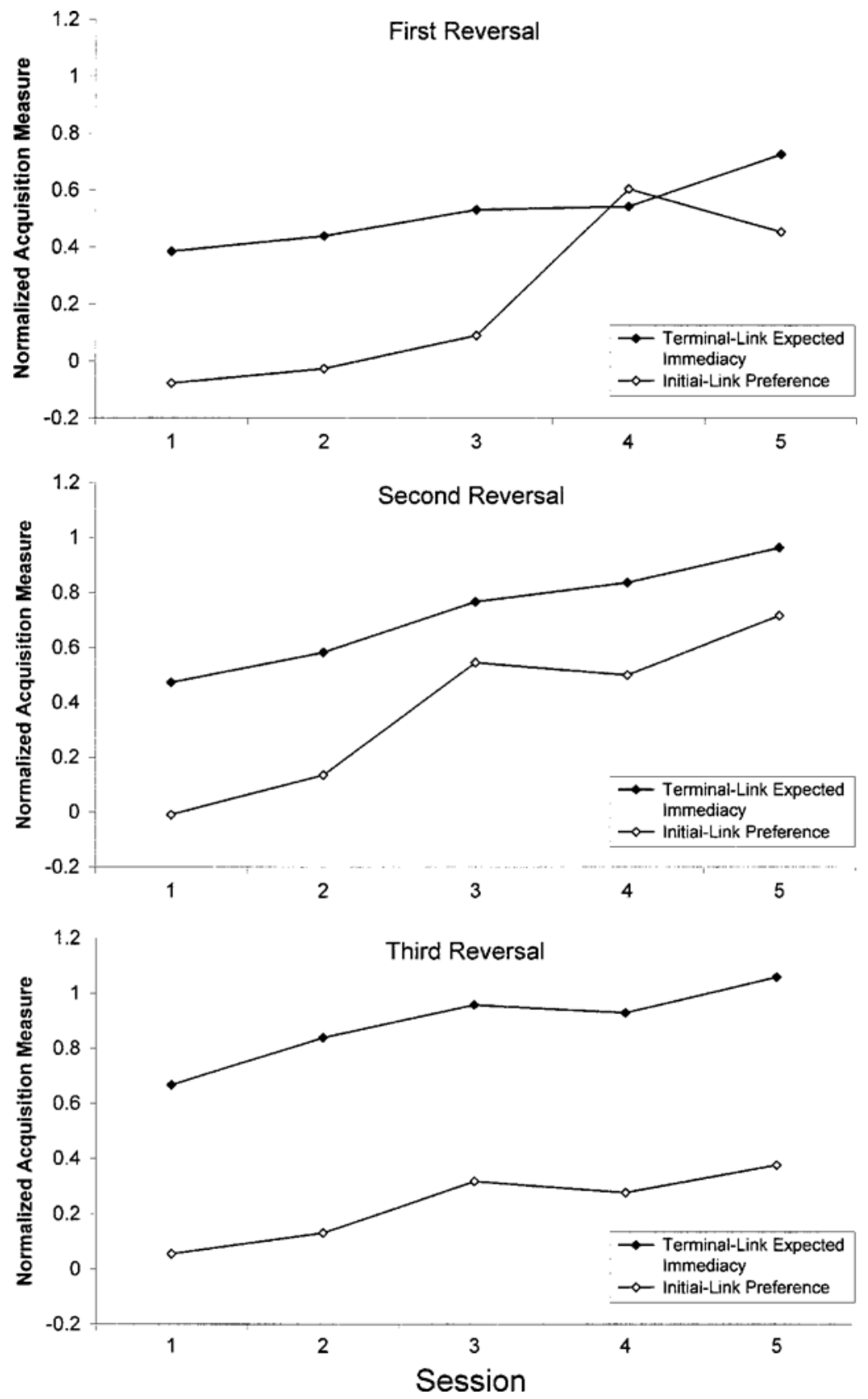

Figure 4. Normalized acquisition data for initial-link preference (unfilled symbols) and terminal-link relative expected immediacy (filled symbols), averaged across subjects. Shown are data from Conditions 2-4 (first, second, and third reversals, respectively) of Experiment 1.

don, 1993). However, the results in Figure 5 are based on a summary statistic (i.e., peak median) that may not be sensitive to all aspects of performance.

Figure 6 shows full response rate distributions for peak trials for individual sessions. Data are shown for asymptotic performance (last session) and the first four post reversal sessions and are averaged across conditions and subjects. Several aspects of Figure 6 are worth noting. Asymptotic data correspond to typical peak-procedure performance, in that response rate distributions are roughly Gaussian, with means at approximately the reinforced durations. At the beginning of the first session, the FI 20 was changed to FI 10 and vice versa, and a dramatic shift in performance occurred. Although the distributions over- 


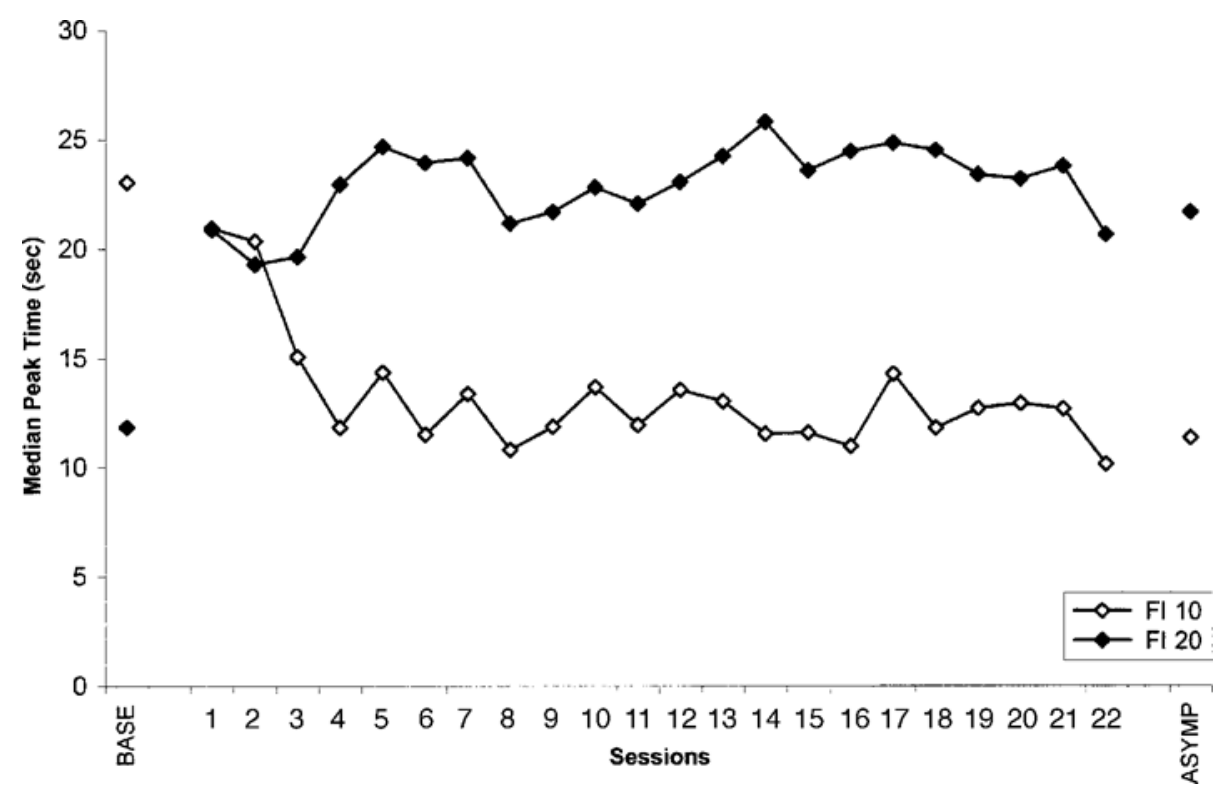

Figure 5. The full course of acquisition for peak medians shown separately for FI 10-sec (unfilled symbols) and FI 20-sec (filled symbols) schedules. The points marked BASE on the abscissa indicate the asymptotes from the previous condition (i.e., baseline), in which the schedules were reversed. Data for the following condition are then shown ( 22 sessions). The points marked ASYMP indicate the average over the last 5 sessions. Data are averaged across conditions and subjects.

lap substantially, a new 10-sec peak has started to develop, and the 10 -sec peak from the previous condition has vanished (as is evidenced by the broad response distribution for FI 20). Across successive sessions, temporal control develops so that by the fourth session, performances that are reasonably close to asymptote have been achieved. However, from Figure 6 there is no clear indication that performance shifts more rapidly for the FI-10-FI-20 transition. Instead, temporal control develops gradually for both schedules. Thus, the evidence in Figure 5 that performance shifted faster for the FI 20 appears to depend on a summary statistic (peak median) that cannot capture all aspects of responding, in particular the strengthening of temporal control across sessions seen in Figure 6.

\section{EXPERIMENT 2}

Successive-reversal designs in which the position of the richer terminal link changes across conditions (e.g., Experiment 1), are very common in the concurrent-chains literature. One advantage is that such designs provide a convenient way to control for response bias during the initial links, since preference between the same pair of terminal links can be averaged across successive conditions (e.g., Moore, 1979). The reversal method used in all previous studies, including Experiment 1, has been to change the terminal-link stimulus-reinforcer relations while preserving the initial-link contingencies. For example, in Figure 1, the terminal-link schedules would be changed so that the red and green center keys are associated with FI $20 \mathrm{sec}$ and
FI $10 \mathrm{sec}$ after the reversal, respectively. But there is another way to implement a reversal: The schedules associated with the different center-key stimuli are maintained while the initial-link keys that produce those stimuli are changed. In other words, after the reversal, the schedules signaled by the red and green center keys remain FI $10 \mathrm{sec}$ and FI 20 sec, but the left initial-link key now leads to green and the right initial-link key leads to red.

The critical difference between the reversal methods is whether the initial- or terminal-link contingencies are altered. If preference in the initial links is mediated by terminal-link value, with value, in turn, depending on the stimulus-reinforcer relations signaled by the terminal links, preference should shift more rapidly if those relations are preserved than if they are switched.

\section{Method}

Subjects. Four White Carneau pigeons, numbered 034, 035, 037, and 283, participated as subjects and were maintained at $85 \%$ of their ad-lib weight $\pm 15 \mathrm{~g}$. All the birds had previous experience with a variety of experimental procedures. They were housed under the same conditions as those in Experiment 1.

Apparatus. The apparatus was the same as that in Experiment 1.

Procedure. Because the subjects were experienced, they were exposed directly to a concurrent-chains procedure that was similar in many respects to the one used in Experiment 1. Sessions consisted of 72 initial- and terminal-link cycles. During the initial links, the side keys were illuminated white, and a terminal-link entry was randomly assigned to either the left or the right key, with the restriction that 18 out of every 36 entries were assigned to each key. A response produced an entry into a terminal link if (1) it was to the preselected key, (2) an interval selected from a VI 15-sec schedule at 

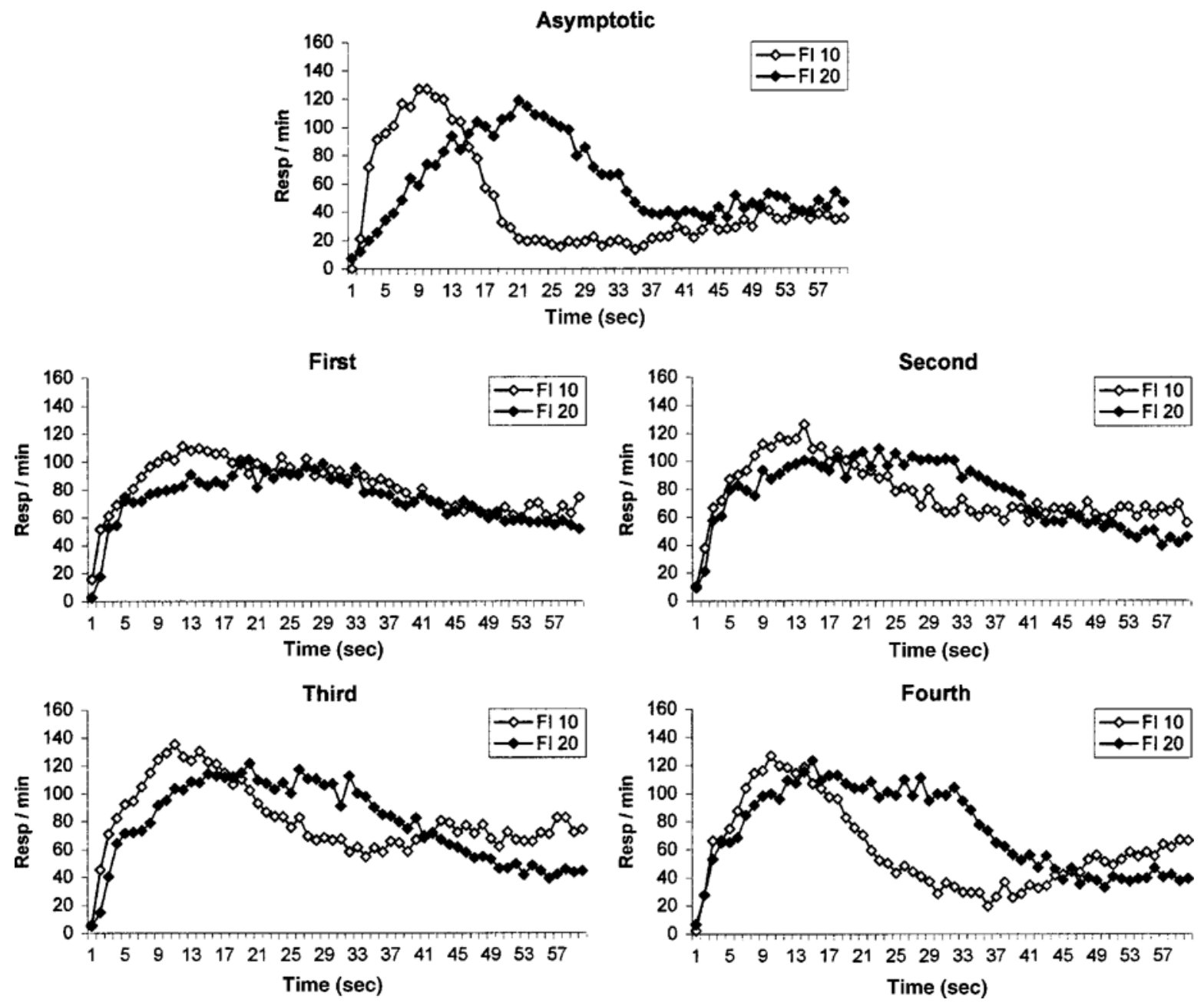

Figure 6. Response rate distributions for peak-trial responding for FI 10-sec (unfilled symbols) and FI 20-sec (filled symbols) schedules. All panels show data from single sessions, averaged across conditions and subjects. Asymptotic data are from the last session of each condition; the other panels represent data from the first four postreversal sessions.

the start of the cycle had timed out, and (3) a 1.5-sec COD was satisfied (i.e., at least $1.5 \mathrm{sec}$ had elapsed following a changeover to the key for which terminal-link entry was arranged). The VI $15 \mathrm{sec}$ contained 18 intervals constructed from an arithmetic progression, $a, a+d, a+2 d, \ldots$, where $a$ equals one eighteenth and $d$ equals one ninth the schedule value. The intervals were sampled randomly without replacement, and separate lists were maintained so that each interval preceded a left and a right terminal-link entry exactly once every 36 cycles.

Entry into a terminal link was signaled by red or green illumination of the center key, coupled with extinguishing of the side keys. The terminal-link schedules were always FI $8 \mathrm{sec}$ and FI $16 \mathrm{sec}$ (assigned to red and green or vice versa, depending on condition). Reinforcement consisted of 2.5 -sec access to grain, during which all lights in the chamber were extinguished. A 5-sec limited hold operated during terminal links, so that if a response had not been made within $5 \mathrm{sec}$ after the FI had elapsed, the terminal link was canceled and the initial links were reinstated. After the terminal-link had finished the side keys were lighted white signaling the initial links and the next cycle began. The houselight remained on at all times, except during reinforcement.
The experiment consisted of a series of reversals in which the position of the initial link leading to the richer terminal link (FI $8 \mathrm{sec}$ ) was changed between conditions. There were two types of reversals, in which the terminal-link stimulus-reinforcer relations (1) were changed (called switched reversals), or (2) were kept constant (consistent reversals). For switched reversals, which is the traditional procedure, the initial-link-terminal-link assignments were kept in place (i.e., the left initial link led to red center key and the right initial link led to the green center key, or vice versa), but the FI schedules associated with the terminal links were changed. For consistent reversals, the red and green center keys signaled the same FI schedules, but the initial-link-terminal-link assignments were changed.

The order of conditions for each subject is listed in Table 2. All the conditions lasted for 20 sessions. Although all the subjects began the experiment at the same time, terminal-link responding for Birds 034, 035, and 037 did not give evidence of the break-run pattern typical of FI performance (Schneider, 1969), likely because these birds (unlike Bird 283) had a recent history of responding to ratio schedules on the center key. To encourage pausing, Birds 034, 035, and 037 were given training under a differential reinforcement of other behavior (DRO) procedure prior to Condition 1. Because 
Table 2

Order of Conditions in Experiment 2

\begin{tabular}{|c|c|c|c|c|c|c|c|c|}
\hline \multirow[b]{2}{*}{ Condition } & \multicolumn{2}{|c|}{ Bird 034} & \multicolumn{2}{|c|}{ Bird 035} & \multicolumn{2}{|c|}{ Bird 283} & \multicolumn{2}{|c|}{ Bird 037} \\
\hline & Schedules & Assign. & Schedules & Assign. & Schedules & Assign. & Schedules & Assign. \\
\hline 1 & FI 16 FI 8 & LR/RG & FI 16 FI 8 & LG/RR & FI 16 FI 8 & LR/RG & FI 8 FI 16 & LG/RR \\
\hline 2 & FI 8 FI $16_{C}$ & LG/RR & FI 8 FI $16_{C}$ & LR/RG & FI 8 FI $16_{s}$ & LR/RG & FI 16 FI $8_{C}$ & LR/RG \\
\hline 3 & FI $16 \mathrm{FI} 8_{\mathrm{S}}$ & LG/RR & FI 16 FI $8_{\mathrm{S}}$ & LR/RG & FI $16 \mathrm{FI} 8_{\mathrm{C}}$ & LG/RR & FI 8 FI $16_{S}$ & $\mathrm{LR} / \mathrm{RG}$ \\
\hline 4 & & & & & FI 8 FI $16_{C}$ & LR/RG & & \\
\hline 5 & & & & & FI $16 \mathrm{FI} 8_{\mathrm{S}}$ & LR/RG & & \\
\hline
\end{tabular}

Note-Listed under "Schedules" are the terminal-link schedules produced by left and right initial-link responses. Listed under "Assign." are the center-key colors associated with the initial links (LR/RG 5 left red, right green; LG/RR 5 left green, right red). Conditions subscripted by "C" designate reversals in which the terminal-link stimulus-reinforcer relations remained consistent; "S" indicates reversals in which the stimulus-reinforcer relations were switched.

of the limited time available for the experiment, these birds were able to complete only one pair of reversals (as compared with two for Bird 283). Thus, the consistent reversal was scheduled first for these birds. In this way, any increase in acquisition rate owing simply to repeated training, which is a commonly finding in discrimination learning (e.g., Beale, 1970; Williams \& Dunn, 1991), would work against the predicted effect.

\section{Results}

The key question in Experiment 2 was whether rate of acquisition of preference for the FI 8-sec terminal link differed between the consistent and the switched conditions. The preference data across the 20 sessions of each condition are shown for individual subjects in Figure 7 (averaged across replications for Bird 283), and group-mean data are shown in the upper panel of Figure 8. Baseline data (i.e., asymptotic preference from the previous condi- tion, expressed as 1 - preference for FI $8 \mathrm{sec}$ ) are also shown. Baseline preference was greater for the consistent condition $(M=0.32)$ than for the switched condition $(M=$ 0.23 ), although the difference was not significant [paired $t(3)=1.09$, n.s.]. Data from the 20 sessions were entered into a repeated measures ANOVA, with condition and session as factors. There were significant main effects of condition $[F(1,3)=12.03]$ and session $[F(19,57)=13.25]$. As the upper panel of Figure 8 shows, overall preference for the FI $8 \mathrm{sec}$ was greater in the consistent condition. The interaction between condition and session was also significant $[F(19,57)=1.92]$. Analysis of simple effects found that preference was significantly greater for the consistent condition in Sessions 2, 3, 10,17, 19, and 20. This interaction is important because it suggests that preference in the consistent condition may have changed more rapidly
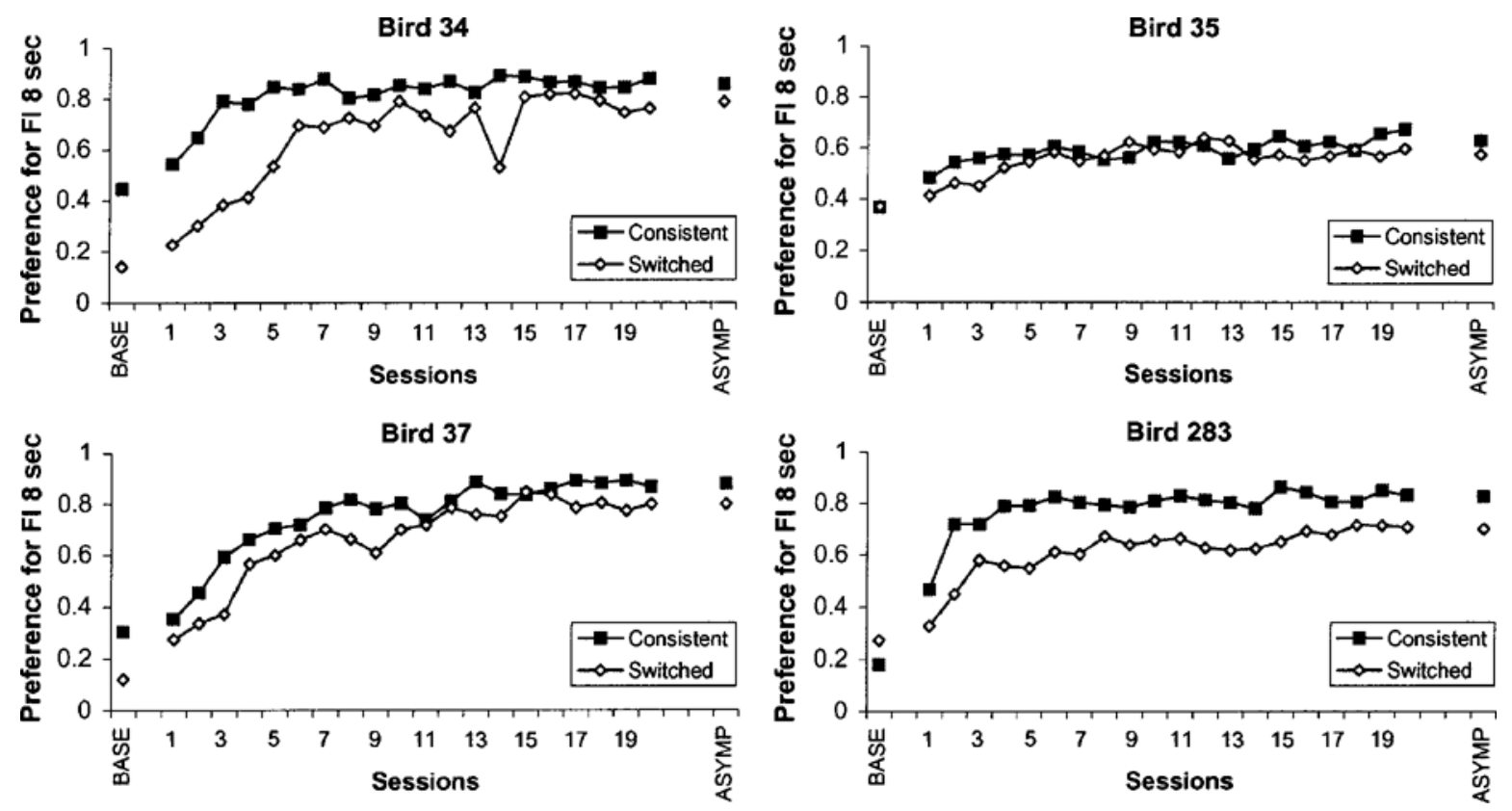

Figure 7. Preference for the FI 8-sec terminal link for individual subjects in Experiment 2. Data are shown for both consistent (filled symbols) and switched (unfilled symbols) conditions. Data points marked BASE represent the asymptotic value from the previous condition (i.e., baseline), in which the terminal links were FI 16 sec FI 8 sec. Data are then shown for the following condition (20 sessions) in which the terminal links were FI 8 sec FI 16 sec. ASY MP indicates asymptotic preference values. 

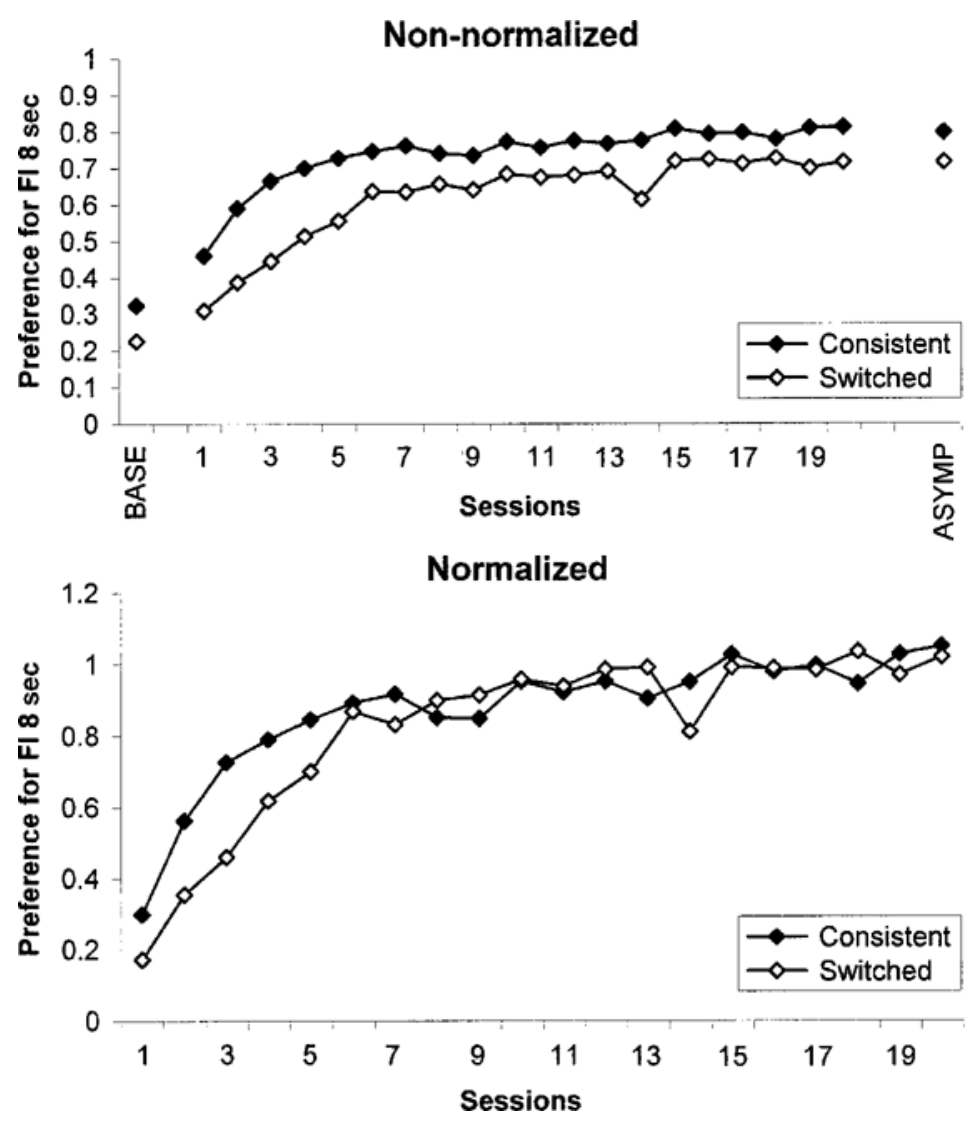

Figure 8. The upper panel shows the average preference data for the consistent (filled symbols) and switched (unfilled symbols) conditions. The lower panel shows the corresponding normalized data. Data were normalized for each subject by expressing preference in each session as a proportion of the difference between baseline and asymptotic values and then were averaged across subjects.

during the early sessions of training. However, this conclusion is complicated by the fact that overall levels of preference were greater in the consistent condition.

We therefore conducted two analyses that controlled for differences in overall levels preference. First, hyperbolic functions were fitted to the group-mean data in Figure 8 and were used to obtain estimates for the number of sessions required to reach $75 \%$ of asymptotic preference. The hyperbolic function (the same as that used in Experiment 1) described the data well, accounting for $95 \%$ and $94 \%$ of the variance in the data from the consistent and the switched conditions, respectively. The estimated number of sessions to $75 \%$ criterion were 3.92 (consistent) and 6.46 (switched).

In the second analysis, the data in Figure 7 were normalized as a proportion of the eventual change in preference during the condition. The lower panel of Figure 8 shows the normalized preference data, averaged across subjects. A repeated measures ANOVA found a main effect of session $[F(19,57)=35.41]$, but not of condition $[F(1,3)=0.92$, n.s. $]$. Importantly, the interaction between condition and session was significant $[F(19,57)=1.81]$.
Analysis of simple effects found that normalized preference for the FI 8 was significantly greater for the consistent condition in Session 3. Figure 8 shows that the difference between the consistent and the switched values was largest in Session 3, although there was a clear tendency for normalized preference to be greater for the consistent condition across the first 5 sessions. Thus, the results from both the hyperbolic fits to the group mean and the analysis of individual normalized data suggest that acquisition of preference was more rapid when the terminallink stimulus-reinforcer relations from the previous condition were maintained (consistent condition), as compared with when they were changed (switched condition). This result supports the hypothesis that relative responding in the initial links depends on terminal-link value.

\section{GENERAL DISCUSSION}

The present experiments used successive-reversal designs to test whether acquisition of choice in concurrent chains would support the view that initial-link preference 
is mediated by the relative values of the terminal links. In one form or another, this value hypothesis has been assumed by all models for concurrent chains (e.g., Davison \& Temple, 1973; Grace, 1994; Killeen, 1982; Mazur, 2001; Squires \& Fantino, 1971; Vaughan, 1985). Since these models have been developed solely on the basis of steady-state data, it is important to test predictions of the value hypothesis regarding acquisition phenomena. The predictions tested here included whether terminal-link responding would change more rapidly than initial-link preference after a reversal (Experiment 1) and whether acquisition of preference would be faster if the terminal-link stimulus-reinforcer relations were maintained (Experiment 2).

Experiment 1 used a combined concurrent-chains/peak procedure (Grace \& Nevin, 1999) with FI 10-sec and FI 20 -sec terminal links, so that measures of the expected reinforcement delays during the terminal links could be obtained in each session, along with choice in the initial links. The relative expected immediacy of reinforcement in the terminal links changed more rapidly than initial-link preference relative to the eventual asymptote, as predicted by the value hypothesis. Experiment 2 compared rate of preference reversals between FI 8-sec and FI 16-sec terminal links when the postreversal stimulus-reinforcer relations were maintained or switched. Preference changed more rapidly when the terminal-link stimulus-reinforcer relations were maintained. The results from both experiments are consistent with the value hypothesis and thus are the first acquisition data to support the basic assumption of concurrent-chains models that responding during the initial links is determined by the relative value of the terminal links.

However, the results from Experiment 1 are based on a measure of relative expected immediacy derived from responding during the no-food terminal-link presentations (i.e., peak trials). This measure assumes that animals' estimate of the time to reinforcement delivery is the median of the peak-trial response distribution. Because the median is a summary statistic, it necessarily omits informationspecifically, that regarding the variability of peak-trial responding. Figure 6 shows that temporal control for FI 10sec and FI 20-sec schedules develops gradually, in terms of a sharpening of the distribution peaks across sessions. It is unclear whether or how this reduction in variability in the response rate distributions-which clearly reflects an acquisition process that continues after the medians have settled down to their asymptotic values - might be incorporated into a summary measure (such as relative expected immediacy) that can be compared with initial-link preference. In any event, various aspects of performance-peak location, variability, and initial-link preference-may be differentially sensitive to the reversal manipulations used here, and this differential sensitivity might be responsible for the faster acquisition rates observed for terminal-link responding, rather than the value hypothesis per se. Nevertheless, Figure 6 shows that there was a dramatic change in the peak-trial distributions during the first session, which may be contrasted with the very small shift $(<0.03)$ in relative initial-link responding (see Figure 3 ). Thus, the prediction of the value hypothesis that terminal-link performance should change more rapidly than initial-linkperformance seems to be supported.

Despite the evidence in favor of the value hypothesis offered here, one important issue that remains unresolved is the time scale over which responding in the initial links is determined by the values of the terminal links. All of the models cited above are molar in the sense that they describe relations between reinforcement parameters and steady-state responding, averaged across sessions at asymptote. Perhaps the simplest version of the value hypothesis requires that choice at the level of individual responses be controlled by terminal-link value. For example, Gibbon et al. (1988) proposed a model for concurrent chains based on scalar expectancy theory (Gibbon, 1977). In their model, subjects are assumed to form separate memorial representations for the reinforcement delays during the terminal links and, during the initial links, to repeatedly sample their memories and to respond to the initial link that leads to the terminal-link stimulus with the shorter remembered delay. According to this view, the acquisition curves should superpose when normalized; a change in relative value should produce an immediate shift in preference.

This simple version of the value hypothesis is challenged by the faster acquisition rates obtained for terminal-link responding in Experiment 1. Our results showed that changes in preference during the initial links lagged equivalent changes in terminal-link responding by approximately 5.5 sessions (see Figure 3 ). If responding during the terminal-link no-food trials is assumed to be the most "direct" measure of stimulus value, this suggests that preference changes fairly sluggishly as a function of value. Any model that seeks to account for concurrent-chains choice in transition, as well as in steady state, will need to explain this finding.

Evidence from prior research that different measures of behavior can be controlled by a single estimate of time to reinforcement is uncertain. Zeiler and Powell (1994) studied the performance of pigeons under the peak procedure, using a wide range of FI schedule values (from 7.5 to $480 \mathrm{sec}$ ). They concluded that the relationship between different measures of behavior and the schedule value depended on the particular behavior considered. For example, peak location was a power function of FI schedule value with an exponent greater than 1.0, but pause durations and acceleration points were linearly related to the schedule value (i.e., an exponent of 1.0). Durlach and Dawson (1991) used a peak procedure, with rats as subjects, to test whether a signaled delay learned for one response (e.g., leverpressing) would transfer to a second response (e.g., chain pulling) trained in the presence of a different signaled delay. If the rats had acquired knowledge of the temporal relations between stimuli and rein- 
forcement independent of the particular responses, transfer should have occurred, whereas complete lack of transfer would indicate that timing performance was specific to the behavioral pattern trained during a particular interval. Durlach and Dawson's data were highly idiosyncratic across subjects and did not clearly support either hypothesis. They suggested that both the explicit stimuli arranged by the experimenters and the manipulandum cues specific to a particular response may acquire control over responding in the peak procedure. In any event, the complexities of the results reported by Zeiler and Powell and by Durlach and Dawson suggest that it may be oversimplifying the situation to assume that a single memorial representation or estimated time to reinforcement can control different aspects of behavior. The results from Experiment 1 are similar, in that independent measures of the same putative construct did not entirely converge, although the procedure was more complex and the phenomena were examined in acquisition as well as in steady state.

The increased rate of acquisition in Experiment 2 during the consistent reversals is similar to the results of Williams (1999). He trained rats on a series of discrimination reversals in which pressing one lever led to food $(\mathrm{S}+)$ after a delay and pressing the other led to no food $(\mathrm{S}-$ ) after the same delay. Different stimulus chains (sequences of visual and auditory stimuli) filled the delay intervals. Williams (1999) found that when the stimulus sequences had the same correlation with food or no-food outcomes as before the reversal, acquisition was faster, as compared with when the correlations were not the same. He interpreted his results as providing support for the view that the value of the stimuli as conditioned reinforcers was a critical determiner of acquisition rate (see also Williams, 1994). Williams's data and ours support the view that terminal-link value is determined by the Pavlovian, stimulus-reinforcer contingency.

Overall, the present experiments provide the first acquisition data to support the view that choice in concurrent chains depends on the learned value of the terminallink stimuli. Acquisition of temporal control of responding during the terminal links occurs more rapidly than corresponding acquisition of initial-link preference, and terminal-link value is determined primarily by the stimulus-reinforcer contingency. An important goal for future research will be to explore relations between initial- and terminal-link responding at the level of individual trials, in an attempt to understand better how choice is mediated by value.

\section{REFERENCES}

Adams, C. D., \& Dickinson, A. (1981). Instrumental responding following reinforcer devaluation. Quarterly Journal of Experimental Psychology, 33B, 109-121.

Alsop, B., \& DAvison, M. (1988). Concurrent-chain performance: Effects of absolute and relative terminal-link entry frequency. Journal of the Experimental Analysis of Behavior, 49, 351-365.

AutoR, S. M. (1960). The strength of conditioned reinforcers as a func- tion of frequency and probability of reinforcement. Unpublished doctoral dissertation, Harvard University.

Beale, I. (1970). The effects of amount of training per reversal on successive reversals of a color discrimination. Journal of the Experimental Analysis of Behavior, 14, 345-352.

Beam, J. J., Killeen, P. R, Bizo, L. A., \& Fetterman, J. G. (1998). How reinforcement context affects temporal production and categorization. Animal Learning \& Behavior, 26, 388-396.

CATANia, A. C. (1970). Reinforcement schedules and psychophysical judgments: A study of some temporal properties of behavior. In W. N. Schoenfeld (Ed.), The theory of reinforcement schedules (pp. 1-42). New York: Appleton-Century-Crofts.

Cheng, K., \& Roberts, W. A. (1991). Three psychophysical principles of timing in pigeons. Learning \& Motivation, 22, 112-128.

Davison, M. C., \& Temple, W. (1973). Preference for fixed-interval schedules: An alternative model. Journal of the Experimental Analysis of Behavior, 20, 393-403.

Durlach, P. J., \& Dawson, G. R. (1991). Response specificity in animal timing. Journal of the Experimental Analysis of Behavior, 55, 11-20.

FAnTINo, E. (1969). Choice and rate of reinforcement. Journal of the Experimental Analysis of Behavior, 12, 723-730.

Gallistel, C. R, \& GibBon, J. (2000). Time, rate, and conditioning. Psychological Review, 107, 289-344.

GibBon, J. (1977). Scalar expectancy theory and Weber's law in animal timing. Psychological Review, 84, 279-325.

Gibbon, J., Church, R. M., Fairhurst, S., \& Kacelnik, A. (1988). Scalar expectancy theory and choice between delayed rewards. Psychological Review, 95, 102-114.

GRACE, R. C. (1994). A contextual model of concurrent-chains choice. Journal of the Experimental Analysis of Behavior, 61, 113-129.

GRACE, R. C. (1996). Choice between fixed and variable delays to reinforcement in the adjusting-delay procedure and concurrent chains. Journal of Experimental Psychology: Animal Behavior Processes, 22, 362-383.

Grace, R. C., \& Nevin, J. A. (1999). Timing and choice in concurrent chains. Behavioural Processes, 45, 115-127.

Grace, R. C., \& Nevin, J. A. (2000). Response strength and temporal control in fixed-interval schedules. Animal Learning \& Behavior, 28, 313-331.

Grace, R. C, \& Savastano, H. I. (2000). Temporal context and conditioned reinforcement value. Journal of Experimental Psychology: General, 129, 427-443.

HERRNSTEIN, R. J. (1964). Secondary reinforcement and rate of primary reinforcement. Journal of the Experimental Analysis of Behavior, 7, 27-36.

HigA, J. J. (1996). Rapid timing of a single transition in interfood interval duration by rats. Animal Learning \& Behavior, 25, 177-184.

Higa, J. J., Thaw, J. M., \& Staddon, J. E. R. (1993). Pigeons' wait-time responses to transitions in interfood-interval duration: Another look at cyclic schedule performance. Journal of the Experimental Analysis of Behavior, 59, 529-541.

KILLEEN, P. (1970). Preference for fixed-interval schedules of reinforcement. Journal of the Experimental Analysis of Behavior, 14, 127-131.

KILlEEN, P. (1982). Incentive theory: II. Models for choice. Journal of the Experimental Analysis of Behavior, 38, 217-232.

MAZUR, J. E. (1992). Choice behavior in transition: Development of preference with ratio and interval schedules. Journal of Experimental Psychology: Animal Behavior Processes, 18, 364-378.

MAzUR, J. E. (2001). Hyperbolic value addition and general models of animal choice. Psychological Review, 108, 96-112.

Moore, J. (1979). Choice and number of reinforcers. Journal of the Experimental Analysis of Behavior, 32, 51-63.

RoBERTS, S. (1981). Isolation of an internal clock. Journal of Experimental Psychology: Animal Behavior Processes, 7, 242-268.

SCHNEIDER, B. A. (1969). A two-state analysis of fixed-interval responding in the pigeon. Journal of the Experimental Analysis of Behavior, 12, 677-687.

Squires, N., \& Fantino, E. (1971). A model for choice in simple concurrent and concurrent-chains schedules. Journal of the Experimental Analysis of Behavior, 15, 27-38. 
Stubbs, D. A., \& Pliskoff, S. S. (1969). Concurrent responding with fixed relative rate of reinforcement. Journal of the Experimental Analysis of Behavior, 12,887-895.

VAUghan, W., JR. (1985). Choice: A local analysis. Journal of the Experimental Analysis of Behavior, 43, 383-405.

Williams, B. A. (1994). Conditioned reinforcement: Neglected or outmoded explanatory construct? Psychonomic Bulletin \& Review, 1, 457-475.

Williams, B. A. (1999). Value transmission in discrimination learning involving stimulus chains. Journal of the Experimental Analysis of Behavior, 72, 177-185.

Williams, B. A., \& Dunn, R. (1991). Substitutability between condi- tioned and primary reinforcers in discrimination acquisition. Journal of the Experimental Analysis of Behavior, 55, 21-35.

Williams, B. A., Ploog, B. O., \& Bell, M. C. (1995). Stimulus devaluation and extinction of chain schedule performance. Animal Learning \& Behavior, 23, 104-114.

Zeiler, M. D., \& Powell, D. G. (1994). Temporal control in fixedinterval schedules. Journal of the Experimental Analysis of Behavior, 61, 1-9.

(Manuscript received November 29, 2000; revision accepted for publication November 12, 2001.) 